\title{
OPEN Energy homeostasis genes modify the association between serum concentrations of IGF-1 and IGFBP-3 and breast cancer risk
}

\author{
Rocío Rodríguez-Valentín ${ }^{1}$, Gabriela Torres-Mejía ${ }^{1 凶}$, Louis Martínez-Matsushita ${ }^{2}$, \\ Angélica Angeles-Llerenas ${ }^{1}$, Liliana Gómez-Flores-Ramos ${ }^{3}$, Roger K. Wolff ${ }^{4}$, \\ Kathy B. Baumgartner ${ }^{5}$, Lisa M. Hines ${ }^{6}$, Elad Ziv ${ }^{7}$, Lourdes Flores-Luna', \\ Luisa Ma. Sánchez-Zamorano ${ }^{1}$, Eduardo Ortiz-Panozo ${ }^{1}$ \& Martha L. Slattery ${ }^{4}$
}

Breast cancer is a multifactorial disease in which the interplay among multiple risk factors remains unclear. Energy homeostasis genes play an important role in carcinogenesis and their interactions with the serum concentrations of IGF-1 and IGFBP-3 on the risk of breast cancer have not yet been investigated. The aim of this study was to assess the modifying effect of the genetic variation in some energy homeostasis genes on the association of serum concentrations of IGF-1 and IGFBP-3 with breast cancer risk. We analyzed 78 SNPs from 10 energy homeostasis genes in premenopausal women from the 4-Corner's Breast Cancer Study (61 cases and 155 controls) and the Mexico Breast Cancer Study (204 cases and 282 controls). After data harmonization, 71 SNPs in HWE were included for interaction analysis. Two SNPs in two genes (MBOAT rs13272159 and NPY rs16131) showed an effect modification on the association between IGF-1 serum concentration and breast cancer risk $\left(P_{\text {interaction }}<0.05\right.$, adjusted $P_{\text {interaction }}<0.20$ ). In addition, five SNPs in three genes (ADIPOQ rs182052, rs822391 and rs7649121, CARTPT rs3846659, and LEPR rs12059300) had an effect modification on the association between IGFBP-3 serum concentration and breast cancer risk $\left(P_{\text {interaction }}<0.05\right.$, adjusted $P_{\text {interaction }}<0.20$ ). Our findings showed that variants of energy homeostasis genes modified the association between the IGF-1 or IGFBP-3 serum concentration and breast cancer risk in premenopausal women. These findings contribute to a better understanding of this multifactorial pathology.

Breast cancer is a multifactorial disease in which the interplay among multiple risk factors remains unclear. Some central and peripheral regulators of food intake and energy expenditure (energy homeostasis) foster cancer development ${ }^{1}$. Dysregulated energetic metabolism has been strongly associated with breast cancer (BC) risk $^{1,2}$. In addition to their role in food intake or fuel metabolism, energy homeostasis factors regulate tumorigenic processes. Central regulators such as the hypothalamic orexigenic neuropeptide Y (NPY) and anorexigenic cocaine and amphetamine regulated transcript (CART) peptide have been involved in different kinds of cancers ${ }^{3,4}$. NPY promotes cellular proliferation, invasion, metastasis and angiogenesis through its different receptors ${ }^{3}$, and the CART peptide is expressed in primary and metastatic $\mathrm{BC}$ cells ${ }^{4}$. Among peripheral regulators, leptin (LEP) signaling stimulates proliferation, survival, migration and cell invasion in $\mathrm{BC}^{5-7}$, and adiponectin (ADIPOQ) inhibits proliferation and metastasis of $\mathrm{BC}$ cells ${ }^{5,6}$. The ghrelin (GHRL) peptide has been associated with increased proliferation of BC cellular lines ${ }^{8}$. The enzyme ghrelin-O-acyltransferase (GOAT or MBOAT4), implicated in the activation of GHRL, is overexpressed in BC tissue samples ${ }^{9}$. Although cholecystokinin (CCK) has not been

\footnotetext{
${ }^{1}$ Center for Population Health Research, National Institute of Public Health, 62100 Cuernavaca, Morelos, Mexico. 'Department of Geriatrics, Hospital General Regional 251, 52148 Metepec, Estado de Mexico, Mexico. ${ }^{3}$ Cátedras CONACYT-Center for Population Health Research, National Institute of Public Health, 62100 Cuernavaca, Morelos, Mexico. ${ }^{4}$ Department of Medicine, University of Utah, Salt Lake City, UT 84108, USA. ${ }^{5}$ Department of Epidemiology and Population Health, School of Public Health and Information Sciences, University of Louisville, Louisville, KY 40202, USA. ${ }^{6}$ Department of Biology, University of Colorado Colorado Springs, Colorado Springs, CO 80918, USA. 'Department of Medicine, Institute of Human Genetics, University of California San Francisco, San Francisco, CA 94158, USA. ${ }^{\circledR}$ email: gtorres@insp.mx
} 
associated with the risk of $\mathrm{BC}$, it has been reported that it affects the proliferation of pancreatic cancer cells ${ }^{10}$. Recently, in the Breast Cancer Health Disparities Study, we found associations between genetic variants of some of these genes and the risk of $\mathrm{BC}^{11}$.

Another key factor associated with BC development is insulin-like growth factor-1 (IGF-1) signaling. IGF-1 regulates fuel metabolism and is an important regulator of cell growth, proliferation, survival, differentiation and cellular transformation in many types of cancer, including $\mathrm{BC}^{12-14}$. Insulin-like growth factor binding protein-3 (IGFBP-3) is the main protein that binds to IGF-1. IGFBP-3, by binding to IGF-1, increases the half-life of circulating IGF-1, modulating its availability and its mitogenic and anti-apoptotic effects ${ }^{13}$. In an independent manner, IGFBP-3 is able to regulate the survival and proliferative activity of healthy and cancer cells ${ }^{15}$. Several studies have shown that IGF-1 serum concentration is positively associated with BC risk ${ }^{16-19}$. The association between the serum concentration of IGFBP-3 and the risk of BC has been inconsistent. Some studies have reported positive associations ${ }^{19-21}$, while others have shown inverse relationships ${ }^{21,22}$. At a genetic level, variants at 2q35 identified by genome wide association studies (GWAS) in European ancestry women may mediate their effect via IGFBP- $5^{23}$.

Based on this evidence, we hypothesized that some energy homeostasis genes may modify the association of IGF-1 and IGFBP-3 with the risk of BC. Therefore, the aim of this study was to assess the modifying effect of genetic variants in 10 energy homeostasis genes (ADIPOQ, CART Prepropeptide (CARTPT), CCK, GHRL, LEP, LEP receptor (LEPR), MBOAT, melanocortin-4-receptor gene (MC4R), NPY and proopiomelanocortin (POMC)) on the association of serum concentrations of IGF-1 and IGFBP-3 with the risk of BC.

\section{Results}

The mean age of premenopausal cases in women from the 4-CBCS and MBCS was 44.3 years and 42.9 years, respectively; the mean age for controls was 42.5 and 42.2, respectively. BMI was higher in controls than in cases and was higher in women from the MBCS than in those from the 4-CBCS. Energy intake was higher in cases than in controls and was higher in women from the 4-CBCS compared with those from the MBCS. Serum concentrations of IGF-1 and IGFBP-3 were higher in cases than in controls in both studies. IGF-1 was higher in both cases and controls in the MBCS; in contrast, IGFBP-3 was higher in both cases and controls in women from the 4-CBCS (Table 1).

For this study, 78 SNPs from 10 energy homeostasis genes were included (Supplementary Table S1); all of them had a minor allele frequency higher than $1 \%$. After data from the 4-CBCS and MBCS populations were harmonized, HWE was tested in controls from both studies. Three SNPs (LEPR rs6673324, and rs1137101; POMC rs6713532) in women from the 4-CBCS (Supplementary Table S2) and four SNPs (CARTPT rsrs2239670; LEPR rs9436739, and rs1938484; NPY rs2023890) from the MBCS were not in HWE (Supplementary Table S3). These SNPs were not included in the analysis. The comparison of genotype frequencies between cases and controls in the 4-CBCS showed statistically significant differences $(P<0.05)$ in two genes: ADIPOQ (rs3821799 and rs1063537) and LEP (rs3828942) (Supplementary Table S2). In the MBCS, differences were observed in LEPR (rs12145690) (Supplementary Table S3). The genotype frequency comparison between studies showed significant differences for SNP rs3774261 in the ADIPOQ gene (Supplementary Table S3).

\section{Energy homeostasis genes modified the association of serum concentrations of IGF-1 and IGFBP-3 with BC risk}

IGF-1. The unadjusted analysis of the association between IGF-1 serum concentration and the risk of BC showed that compared to women in the lowest tertile, those in the highest tertile had a statistically significant increased risk of $\mathrm{BC}(\mathrm{OR}=2.79 ; 95 \% \mathrm{CI} 1.88,4.13)$ (Table 2$)$.

We assessed the interaction between IGF-1 and the 71 SNPs and found an effect modification with a $P_{\text {interaction }}<0.05$ for four SNPs in four genes (GHRL, LEPR, MBOAT, and NPY) and with a $P_{\text {interaction }}$ from 0.05 to $<0.10$ for three SNPs in three genes (ADIPOQ, LEPR, and POMC) in co-dominant models (Table 2). When comparing the effect of the highest IGF-1 tertile vs. the lowest, for most SNPs, there was a protective effect in heterozygous women; these effects were statistically significant for ADIPOQ rs17366568 (OR = 0.09; 95\% CI 0.01, 0.91; $P=0.04$ ), and POMC rs7565427 (OR=0.05; 95\% CI 0.01, 0.51; $P=0.01$ ) (Table 2). Of particular interest, the SNP rs13272159 of MBOAT4 showed an increased risk when comparing the effect of the highest IGF-1 tertile vs. the lowest $(\mathrm{OR}=7.16$; $95 \% \mathrm{CI} 1.64,31.25 ; P=0.009)$ in women with AA; the SNP rs7565427 of POMC also showed an increased risk $(\mathrm{OR}=1.89$; $95 \% \mathrm{CI} 1.07,3.35 ; P=0.03)$ in women with GG. After adjusting for multiple comparisons (FDR correction), only interactions for three SNPs in three genes (LEPR rs12059300, MBOAT rs13272159 and NPY rs16131) were statistically significant with an adjusted $P_{\text {interaction }}<0.20$.

After assesing the association between the Table 2 SNPs and IGF-1 serum concentration, we found that for the LEPR rs970468 SNP, control women who were carriers of the GG variant had higher IGF-1 serum concentration $(1.23 \mathrm{ng} / \mathrm{mL}, P=0.010)$ than TT homozygotes. However, after adjusting for multiple comparisons, this association was not statistically significant (adjusted $P>0.05$ ) (data not shown).

IGFBP-3. The unadjusted analysis of the association between IGFBP-3 serum concentration and the risk of $\mathrm{BC}$ showed that compared to women in the lowest tertile, those in the highest tertile had a statistically significant increased risk of $\mathrm{BC}(\mathrm{OR}=5.55,95 \%$ CI $3.55,8.68)$ (Table 3$)$.

For IGFBP-3, we also assessed the interaction with the 71 SNPs and found an effect modification with a $P_{\text {interaction }}<0.05$ for five SNPs in three genes $(A D I P O Q, C A R T P T$ and $L E P R)$ and with a $P_{\text {interaction }}$ from 0.05 to $<0.10$ for five SNPs in three (ADIPOQ, $L E P R$, and NPY) out of the 10 genes included in the analysis (Table 3). In the codominant models, all SNPs except three (ADIPOQ rs182052, and rs7649121; and LEPR rs11585329) showed the same pattern. For seven SNPs (ADIPOQ rs822391, rs3821799, and rs3774261; CARTPT rs3846659; 


\begin{tabular}{|c|c|c|c|c|}
\hline \multirow[b]{3}{*}{ Characteristics } & \multicolumn{2}{|c|}{$\begin{array}{l}\text { 4-Corner's Breast Cancer Study } \\
\text { (4-CBCS) }\end{array}$} & \multicolumn{2}{|c|}{$\begin{array}{l}\text { Mexico Breast Cancer Study } \\
\text { (MBCS) }\end{array}$} \\
\hline & Control & Case & Control & Case \\
\hline & $n=155$ & $n=61$ & $\mathrm{n}=\mathbf{2 8 2}$ & $n=204$ \\
\hline Age $\left(\right.$ years) ${ }^{\mathrm{a}}$ & $42.5(6.5)$ & $44.3(5.0)$ & $42.2(4.5)$ & $42.9(5.1)$ \\
\hline Age at menarche (years) ${ }^{\mathrm{a}}$ & $12.8(1.6)$ & $13.1(1.7)$ & $12.7(1.7)$ & $12.7(1.5)$ \\
\hline 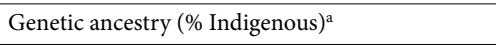 & $19.7(22.6)$ & $16.3(22.9)$ & $71.1(17.4)$ & $69.1(18.0)$ \\
\hline BMI $(\mathrm{Kg} / \mathrm{m} 2)^{\mathrm{a}}$ & $28.4(6.7)$ & $26.4(6.4)$ & $30.0(5.4)$ & $28.3(4.9)$ \\
\hline Height $(\mathrm{cm})^{\mathrm{a}}$ & $161.9(7.3)$ & $161.7(7.5)$ & $153.4(5.5)$ & $154.5(5.5)$ \\
\hline Energy intake (Kcal/day) $)^{a}$ & $2322.5(994.7)$ & $2500.4(1092.9)$ & $2023.4(729.6)$ & $2232.7(755.0)$ \\
\hline IGF-1 serum concentration $(\mathrm{ng} / \mathrm{mL})^{\mathrm{a}}$ & $145.9(50.0)$ & $152.3(42.9)$ & $171.2(98.7)$ & $224.7(101.5)$ \\
\hline IGFBP-3 serum concentration $(\mathrm{ng} / \mathrm{mL})^{\mathrm{a}}$ & $4241.4(761.6)$ & $4455.0(611.2)$ & $3158.3(999.5)$ & $4160.5(1153.4)$ \\
\hline Ever use Oral Contraceptives (yes) ${ }^{\mathrm{b}}$ & 69.7 & 78.0 & 53.9 & 57.4 \\
\hline Ever alcohol consumption (yes) ${ }^{\mathrm{b}}$ & 41.8 & 47.5 & 1.8 & 3.9 \\
\hline 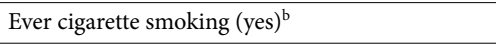 & 28.4 & 31.2 & 22.7 & 27.9 \\
\hline First-degree family history of breast cancer (yes) ${ }^{b}$ & 16.1 & 18.3 & 4.3 & 5.4 \\
\hline \multicolumn{5}{|l|}{ Parity $^{\mathrm{b}}$} \\
\hline Nulliparous & 18.1 & 16.4 & 7.1 & 5.9 \\
\hline 1 or 2 & 43.9 & 47.5 & 40.1 & 45.1 \\
\hline 3 or more & 38.1 & 36.1 & 52.8 & 49.0 \\
\hline \multicolumn{5}{|l|}{ Education $^{\mathrm{b}}$} \\
\hline Low & 7.1 & 4.9 & 85.5 & 76.5 \\
\hline Middle & 17.4 & 18.0 & 8.2 & 13.2 \\
\hline High & 75.5 & 77.1 & 6.4 & 10.3 \\
\hline \multicolumn{5}{|l|}{ IGF-1 serum concentration $(\mathrm{ng} / \mathrm{mL})^{\mathrm{b}} \mathrm{c}$} \\
\hline Tertile 1 & 33.6 & 27.9 & 33.3 & 17.2 \\
\hline Tertile 2 & 33.6 & 31.2 & 33.7 & 25.0 \\
\hline Tertile 3 & 32.9 & 41.0 & 33.0 & 57.8 \\
\hline \multicolumn{5}{|l|}{ IGFBP-3 serum concentration $(\mathrm{ng} / \mathrm{mL})^{\mathrm{b}} \mathbf{d}$} \\
\hline Tertile 1 & 33.6 & 21.3 & 33.3 & 8.8 \\
\hline Tertile 2 & 33.6 & 34.4 & 33.3 & 29.4 \\
\hline Tertile 3 & 32.9 & 44.3 & 33.3 & 70.6 \\
\hline
\end{tabular}

Table 1. Subject characteristics by study (premenopausal women), the Breast Cancer Health Disparities Study. ${ }^{a}$ Mean and standard deviation (SD). ${ }^{b}$ Percentage. ${ }^{c}$ Cut points of tertiles of serum IGF-1 in 4-Corner's Breast Cancer Study were (122.0, 161.0); in Mexico Breast Cancer Study $(123.6,200.7) .{ }^{\mathrm{d}}$ Cut points of tertiles of serum IGFBP-3 1 in 4-Corner's Breast Cancer Study were (3916, 4490); in Mexico Breast Cancer Study (2744, 3535).

LEPR rs12059300; NPY rs16141 and rs16129), when comparing the effect of the highest IGFBP-3 serum concentration tertile vs. the lowest, there was a statistically significant increased BC risk in those who were homozygous for the major allele. This effect was lower in heterozygous, and the effect was not observed in women who were homozygous for the minor allele. For example, for ADIPOQ rs3774261 GG genotype, we observed an $\mathrm{OR}=10.80$ (95\% CI 3.74, 31.19; $P<0.001)$; for the GA genotype, an OR=3.81 (95\% CI 1.75, 8.27; $P=0.001)$; and for the AA genotype, an OR $=2.24$ (95\% CI 0.57, 8.88; $P=0.25)$ (Table 3). All interactions except (LEPR rs11585329) remained statistically significant with an adjusted $P_{\text {interaction }}<0.20$ after adjusting for multiple comparisons (FDR correction).

After assessing the association between the Table 3 SNPs and IGFBP-3 serum concentration, we found that in control women, there was an association between 4 ADIPOQ SNPs (rs7649121, rs182052, rs3774261, rs3821799) and the IGFBP-3 serum concentration $(P<0.05)$. However, after adjusting for multiple comparisons, only the association for rs7649121 remained statistically significant. Compared to AA homozygotes TT carriers had a lower IGFBP-3 serum concentration $(-710.1 \mathrm{ng} / \mathrm{mL})(P=<0.001)$ (data not shown).

\section{Discussion}

In this study, we showed that variants of some energy homeostasis genes modified the association between IGF-1 or IGFBP-3 serum concentration and the risk of BC in premenopausal women. Several epidemiological studies have highlighted the existing association between IGF-1 and IGFBP-3 serum concentration and the risk of $\mathrm{BC}^{12,16,19,21,22}$. IGF-1 and IGFBP-3 regulate multiple biological mechanisms, including fuel metabolism and cell growth, proliferation, migration, metastasis and angiogenesis ${ }^{15}$, 24 . IGF-1 activates downstream pathways involved in carcinogenic processes such as the PI3K/AKT, RAS/RAF/MAPK, and STAT cascades ${ }^{24}$. IGFBP-3, in addition to modulating IGF-1 activity, may also trigger IGF-independent activation of various signaling pathways, 


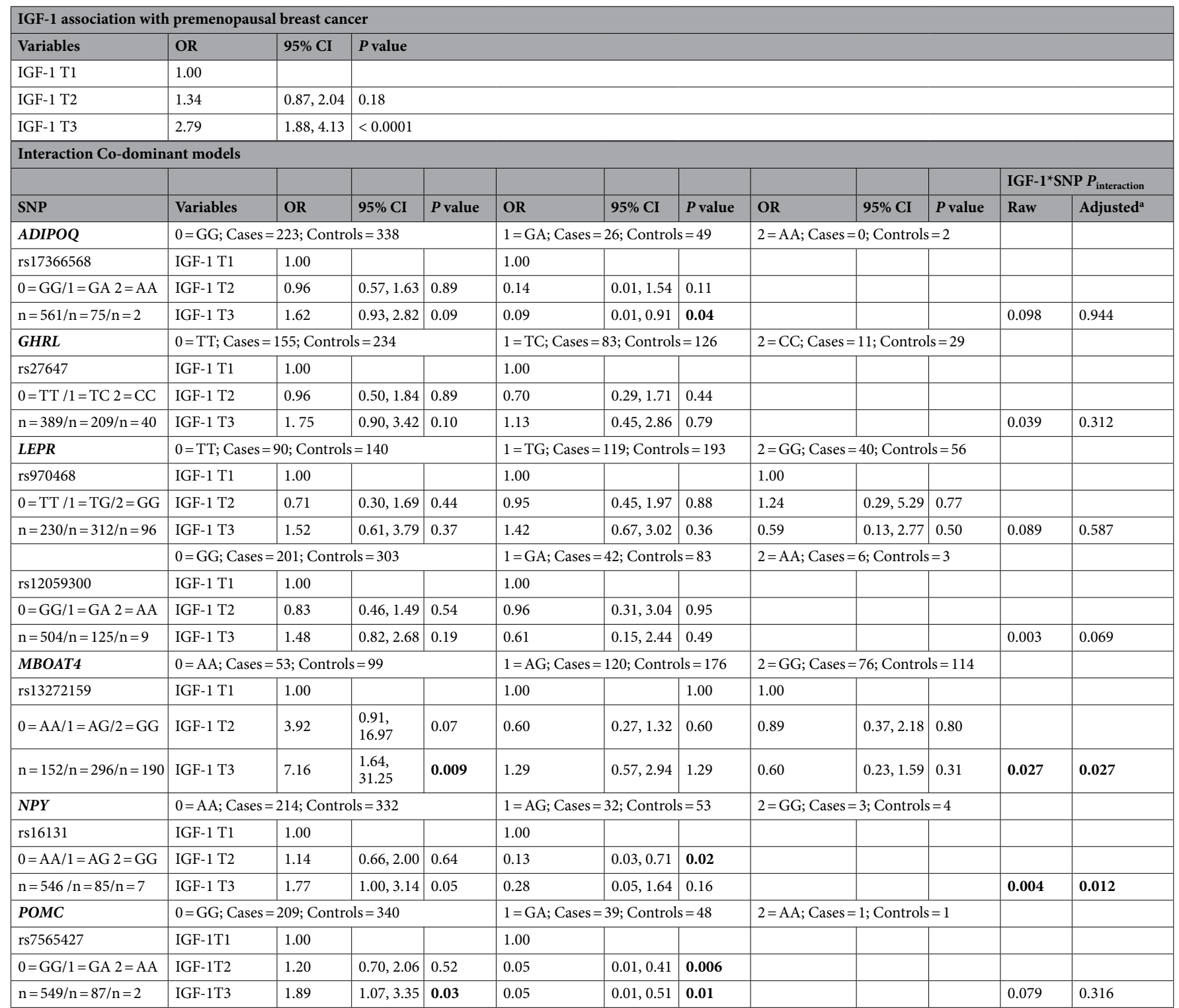

Table 2. Association between serum concentration of IGF-1 and breast cancer by SNPs of genes regulating energy homeostasis, the Breast Cancer Health Disparities Study. The model at the top of the table was not adjusted for potential confounders. The rest of the models were adjusted for IGFBP-3 serum concentration $(\mathrm{ng} / \mathrm{mL})$, age (years), genetic ancestry (\% Indigenous), BMI $(\mathrm{Kg} / \mathrm{m} 2)$, energy intake $(\mathrm{Kcal} /$ day), height $(\mathrm{cm})$, age at menarche (years), ever use oral contraceptives (no,yes), parity (nulliparous, 1 or 2, 3 or more), study (4-CBCS, MBCS), first-degree family history of breast cancer (no, yes), ever alcohol consumption (no, yes) and ever cigarette smoking (no, yes). ${ }^{a}$ Benjamini-Hochberg adjusted $P$ values (correction for the number of SNPs tested per gene); a threshold of 0.2 was considered significant.

including MAPK, AKT, Smad and STAT ${ }^{15,25}$. Recently, we reported an association between some SNPs of energy homeostasis genes and the risk ${ }^{11}$ or mortality ${ }^{26}$ of BC. Other authors have also reported associations between variants of these genes and $\mathrm{BC}$ risk $^{27-29}$. As we described below, evidence shows that some energy homeostasis genes may also regulate signaling pathways involved in cancer development, supporting their potential to modify the association between IGF-1 and IGFBP-3 and the risk of BC.

In our study, seven SNPs in five genes (MBOAT4 rs13272159, NPY rs16131, ADIPOQ rs182052, rs822391 and rs7649121, CARTPT rs3846659, and LEPR rs12059300) showed a significant modifying effect $\left(P_{\text {interaction }}<0.05\right.$; adjusted $\left.P_{\text {interaction }}<0.20\right)$, with at least one tertile of IGF-1 or IGFBP-3 associated with the risk of BC $(P$ value $<0.05)$.

We found that one SNP of MBOAT4 significantly modified the association of IGF-1 serum concentration and the risk of BC in premenopausal women. For the intronic (3'UTR) SNP of MBOAT4, rs13272159, the positive effect of the highest tertile of IGF-1 on the risk of BC was observed in women with the AA genotype but not in 


\begin{tabular}{|c|c|c|c|c|c|c|c|c|c|c|c|c|}
\hline \multicolumn{13}{|c|}{ IGFBP-3 association with premenopausal breast cancer } \\
\hline Variables & OR & $95 \% \mathrm{CI}$ & \multicolumn{10}{|l|}{$P$ value } \\
\hline IGFBP-3 T2 & 2.03 & $1.25,3.31$ & \multicolumn{10}{|l|}{0.004} \\
\hline \multicolumn{13}{|c|}{ Interaction Co-dominant models } \\
\hline & & & & & & & & & & & \begin{tabular}{|l|l|}
$\begin{array}{l}\text { IGF-1 }{ }^{*} \text { SNPP } \\
\text { interaction }\end{array}$ \\
\end{tabular} & \\
\hline rs182052 & IGFBP-3 T1 & 1.00 & & & 1.00 & & & 1.00 & & & & \\
\hline $0=\mathrm{GG} / 1=\mathrm{GA} / 2=\mathrm{AA}$ & IGFBP-3 T2 & 0.69 & $0.23,2.07$ & 0.51 & 3.11 & $1.39,6.97$ & 0.006 & 1.91 & $0.46,7.93$ & 0.37 & & \\
\hline \multirow[t]{2}{*}{$\mathrm{n}=168 / \mathrm{n}=322 / \mathrm{n}=138$} & IGFBP-3 T3 & 1.45 & $0.52,4.08$ & 0.48 & 4.92 & $2.15,11.23$ & $<0.001$ & 16.52 & $3.94,69.26$ & $<0.001$ & 0.027 & 0.164 \\
\hline & \multicolumn{4}{|c|}{$0=$ TT; Cases $=173 ;$ Controls $=262$} & \multicolumn{3}{|c|}{$1=\mathrm{TC} ;$ Cases $=70 ;$ Controls $=113$} & \multicolumn{3}{|c|}{$2=\mathrm{CC} ;$ Cases $=6 ;$ Controls $=14$} & & \\
\hline rs822391 & IGFBP-3 T1 & 1.00 & & & 1.00 & & & & & & & \\
\hline $0=\mathrm{AA} / 1=\mathrm{AT} 2=\mathrm{TT}$ & IGFBP-3 T2 & 1.51 & $0.73,3.13$ & 0.27 & 1.65 & $0.66,4.17$ & 0.29 & & & & & \\
\hline \multirow[t]{2}{*}{$\mathrm{n}=345 / \mathrm{n}=244 / \mathrm{n}=49$} & IGFBP-3 T3 & 3.23 & $1.54,6.77$ & 0.002 & 5.63 & $2.28,13.88$ & $<0.001$ & & & & 0.036 & 0.164 \\
\hline & \multicolumn{4}{|c|}{$0=$ CC $;$ Cases $=78 ;$ Controls $=116$} & \multicolumn{3}{|c|}{$1=$ CT; Cases $=120 ;$ Controls $=191$} & $2=\mathrm{TT}$ & $=51 ;$ Contro & & & \\
\hline rs3821799 & IGFBP-3 T1 & 1.00 & & & 1.00 & & & 1.00 & & & & \\
\hline $0=\mathrm{CC} / 1=\mathrm{CT} / 2=\mathrm{TT}$ & IGFB-P3 T2 & 2.12 & $0.71,6.30$ & 0.18 & 1.15 & $0.52,2.55$ & 0.73 & 2.92 & $0.86,9.89$ & 0.09 & & \\
\hline $\mathrm{n}=194 / \mathrm{n}=311 / \mathrm{n}=133$ & IGFBP-3 T3 & 11.73 & $3.72,36.9$ & $<0.001$ & 3.23 & $1.50,6.93$ & 0.003 & 3.22 & $0.88,11.74$ & 0.08 & 0.078 & 0.187 \\
\hline & $0=\mathrm{GG} ;$ Cases & $=82$; Contro & $s=131$ & & $1=\mathrm{G}$ & $=119$; Contro & 184 & $2=\mathrm{AA}$ & $=48 ;$ Contr & & & \\
\hline rs3774261 & IGFBP-3 T1 & 1.00 & & & 1.00 & & & 1.00 & & & & \\
\hline $0=\mathrm{GG} / 1=\mathrm{GA} / 2=\mathrm{AA}$ & IGFBP-3 T2 & 2.32 & $0.82,6.59$ & 0.11 & 1.38 & $0.61,3.09$ & 0.44 & 2.30 & $0.64,8.18$ & 0.20 & & \\
\hline $\mathrm{n}=213 / \mathrm{n}=303 / \mathrm{n}=121$ & IGFBP-3 T3 & 10.80 & $3.74,31.19$ & $<0.001$ & 3.81 & $1.75,8.27$ & 0.001 & 2.24 & $0.57,8.88$ & 0.25 & 0.057 & 0.171 \\
\hline CARTPT & $0=\mathrm{GG} ;$ Cases & $=198 ;$ Contr & $\mathrm{s}=304$ & & $1=\mathrm{G}$ & $=45$; Control & & $2=C C$ & $=6 ;$ Contro & & & \\
\hline rs11585329 & IGFBP-3 T1 & 1.00 & & & 1.00 & & & & & & & \\
\hline $0=\mathrm{GG} / 1=\mathrm{GT} 2=\mathrm{TT}$ & IGFBP-3 T2 & 1.94 & $1.07,3.51$ & 0.03 & 0.77 & $0.14,4.19$ & 0.76 & & & & & \\
\hline $\mathrm{n}=505 / \mathrm{n}=120 / \mathrm{n}=13$ & IGFBP-3 T3 & 4.12 & $2.28,7.45$ & $<0.001$ & 8.70 & $1.74,43.56$ & 0.008 & & & & 0.075 & 0.849 \\
\hline$N P Y$ & $0=\mathrm{CC} ;$ Cases & $=102 ;$ Contr & $l s=165$ & & $1=\mathrm{C}$ & $=110$; Contro & 169 & $2=\mathrm{TT}$ & $=31$; Contro & & & \\
\hline rs16141 & IGFBP-3 T1 & 1.00 & & & 1.00 & & & 1.00 & & & & \\
\hline $0=\mathrm{CC} / 1=\mathrm{CA} / 2=\mathrm{AA}$ & IGFBP-3 T2 & 2.05 & $0.79,5.36$ & 0.14 & 1.72 & $0.75,3.95$ & 0.20 & 0.86 & $0.20,3.81$ & 0.85 & & \\
\hline $\mathrm{n}=267 / \mathrm{n}=279 / \mathrm{n}=92$ & IGFBP-3 T3 & 6.8 & $2.51,18.4$ & $<0.001$ & 4.48 & $1.96,10.24$ & $<0.001$ & 1.61 & $0.41,6.30$ & 0.50 & 0.056 & 0.116 \\
\hline & $0=\mathrm{GG}$; Cases & $=110 ;$ Contr & $\mathrm{ls}=185$ & & $1=G$ & $=108 ;$ Contro & 156 & $2=\mathrm{TT}$ & $=31 ;$ Contro & & & \\
\hline rs16129 & IGFBP-3 T1 & 1.00 & & & 1.00 & & & 1.00 & & & & \\
\hline $0=\mathrm{GG} / 1=\mathrm{GT} / 2=\mathrm{TT}$ & IGFBP-3 T2 & 2.23 & $0.89,5.58$ & 0.09 & 1.43 & $0.62,3.27$ & 0.40 & 1.09 & $0.21,5.68$ & 0.92 & & \\
\hline $\mathrm{n}=295 / \mathrm{n}=264 / \mathrm{n}=79$ & IGFBP-3 T3 & 6.40 & $2.52,16.25$ & $<0.001$ & 3.75 & $1.64,8.56$ & 0.002 & 2.41 & $0.58,10.4$ & 0.23 & \begin{tabular}{|l|l|l|l|l}
0.077 & \\
\end{tabular} & 0.116 \\
\hline
\end{tabular}

Table 3. Association between serum concentration of IGFBP-3 and breast cancer by SNPs of genes regulating energy homeostasis, the Breast Cancer Health Disparities Study. The model at the top of the table was not adjusted for potential confounders. The rest of the models were adjusted for IGF-1 serum concentration (ng/ $\mathrm{mL})$, age (years), genetic ancestry (\% Indigenous), BMI $(\mathrm{Kg} / \mathrm{m} 2)$, energy intake (Kcal/day), height $(\mathrm{cm})$, age at menarche (years), ever use oral contraceptives (no,yes), parity (nulliparous, 1 or 2, 3 or more), study (4-CBCS, MBCS), first-degree family history of breast cancer (no, yes), ever alcohol consumption (no, yes) and ever

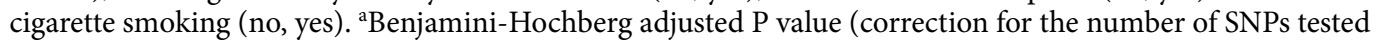
per gene); a threshold of 0.2 was considered significant. 
the presence of the $\mathrm{G}$ allele. There is evidence that supports the possible role of MBOAT4 in BC. The MBOAT4 gene encodes GHRL O-acyltransferase (GOAT), an enzyme that catalyzes GHRL octanoylation regulating GHRL activity $^{30}$. The regulation of growth hormone $(\mathrm{GH})$ and IGF-1 release is under the influence of GHRL ${ }^{27,30}$. In our study, we did not find an association between the MBOAT4 rs13272159 SNP and the serum concentration of IGF-1 (data not shown). The peptide hormone GHRL, in addition to having a role in the regulation of feeding and energy balance, also regulates processes associated with cancer, such as cell proliferation, apoptosis, cell migration, cell invasion, angiogenesis and inflammation. The GHRL proliferation effect could be mediated through the activation of key signaling pathways, such as MAPK/ERK and/or PI3K/AKT/mTOR ${ }^{8,9,30}$. Although a precise role for GHRL in BC has not yet been established, it has been suggested that an imbalance in the expression of the GHRL system in mammary tissue could be implicated in breast tumor pathogenesis ${ }^{9}$. MBOAT4 mRNA expression seems to be upregulated in BC tumors ${ }^{9}$. Our group and other authors have reported an association between GHRL SNPs and BC risk ${ }^{11,28,31}$ or BC-specific mortality ${ }^{26}$. In addition, in a recent cohort study, GHRL expression was associated with increased survival in node-negative patients ${ }^{32}$.

For the intronic SNP of NPY, rs16131, the highest tertile of IGF-1 concentration was borderline associated with an increased risk of BC in the presence of AA but not in G carriers. The central orexigenic regulator NPY and its receptors have been implicated as growth-promoting factors in various cancer types. It has been shown that NPY regulates proliferation, migration, and vascular endothelial growth factor (VEGF) release to promote angiogenesis in BC cells. The mitogenic effects of NPY seem to be mediated by the p44/42 MAPK pathway in some malignancies ${ }^{3}$. It has been suggested that NPY may influence GH release (by modulating growth hormonereleasing hormone $(\mathrm{GHRH})$ secretion $)^{33}$, thus modulating IGF-1 release. There was no association between the NPY rs16131 SNP and the serum concentration of IGF-1 (data not shown). NPY receptor type 1 (NPY1R) has been proposed as a novel peripheral blood marker predictive of metastasis and prognosis in patients with $\mathrm{BC}^{34}$. In a previous work, we found that another NPY SNP, rs16129, was significantly associated with BC-specific mortality; however, no effect was observed for the rs16131 SNP26.

Our results showed that the association between IGFBP-3 serum concentration and BC risk differed by ADIPOQ SNPs (rs822391, rs7649121 and rs182052). In the case of ADIPOQ SNP rs822391, an increase in BC risk was observed for the highest tertile of serum concentration of IGFBP-3 in TT carriers and was lost in the presence of the minor allele C. Interestingly, other authors have reported that rs822391 (minor allele C) decreases the risk of overall prostate cancer ${ }^{35}$. Regarding the $A D I P O Q$ rs7649121 SNP, compared to women in the lowest IGFBP-3 serum concentration tertile, women in the highest tertile showed higher BC risk. This association was higher in carriers of the T allele, than in AA homozygotes. To our knowledge, no association between this SNP and the risk of any cancer has been previously reported. For ADIPOQ SNP rs182052, our data showed that the positive effect of the highest tertile of IGFBP-3 concentration on the risk of BC was higher in the presence of the minor allele A. These results are consistent with other studies that have found that rs182052 (minor allele A) increased the risk of other types of cancer $^{35,36}$. Furthermore, these studies observed an association between rs182052 (minor allele A) and low serum and plasma ADIPOQ concentrations $s^{35,36}$, suggesting a biological causal link. Epidemiological studies have found that ADIPOQ plasma concentrations are inversely associated with the risk of several malignancies, including $\mathrm{BC}^{7}$. It has been suggested that $\mathrm{ADIPOQ}$ is a $\mathrm{BC}$ malignant progression and prognosis biomarker ${ }^{6}$. Research on ADIPOQ has provided evidence for the pleiotropic role of this hormone; it regulates insulin-sensitizing, inflammation, and tumoral actions ${ }^{37}$. Some studies have shown that ADIPOQ regulates GH secretion from rat pituitary cells in vitro, both positively ${ }^{38}$ and negatively ${ }^{39}$. In addition, ADIPOQ receptors are expressed in human pituitary GH-producing cells ${ }^{40}$, suggesting that alterations in ADIPOQ expression could affect GH serum concentration and consequently IGF-1 and IGFBP-3 release. In our study, there was a statistically significant association between the ADIPOQ rs7649121 SNP and a lower serum concentration of IGFBP-3. The ADIPOQ induces cell growth arrest and apoptosis and suppresses cell proliferation, invasion and migration in ER-negative BC cells, whereas controversial observations have been reported for ER-positive BC cells $s^{5}$. ADIPOQ is known to block proliferative signals of IGF-1 and LEP ${ }^{6}$. ADIPOQ signaling induces AMPK activation and consequent inhibition of the MAPK, PI3K/Akt, WNT- $\beta$-catenin, NFkB, and JAK2/STAT3 pathways $^{5,7}$. Given that the ADIPOQ rs7649121 SNP was associated with the IGFBP-3 serum concentration it is not possible to disentangle if the modifying effect was due to a breast cancer signaling pathway or to the effect of the SNP on the IGFBP-3 serum concentration.

We also found an effect modification on the association between the IGFBP-3 serum concentration and the risk of BC by LEPR SNP (rs12059300). Our results show that the positive effect of the IGFBP-3 highest serum concentration tertile on the BC risk observed in women with the GG decreased in the presence of the minor allele A. Interestingly, it has been reported that rs 12059300 interacts with methylation sites in the 5'UTR of $L E P R$, influencing LEP serum levels (LEP levels decrease for AA compared to the GG genotype) ${ }^{41}$. Some studies have observed an association between high LEP plasma concentrations and BC risk ${ }^{5,7}$. High LEP serum concentrations and LEPR overexpression have been positively correlated with the reduction in overall survival rates in patients with $\mathrm{BC}^{6}$. In a previous work, using the samples of the present study plus samples from other centers, we found that some LEPR SNPs were associated with BC risk ${ }^{11}$ and with BC mortality ${ }^{26}$ within specific ancestry strata; however, there was no association between rs 12059300 and BC risk or mortality ${ }^{11,26}$. Experimental data obtained with LEPR knockout mice suggested that LEP signaling could induce GH release ${ }^{42}$, thus regulating not only IGF-1 but also IGFBP-3 release. We did not find an association between the LEPR rs12059300 SNP and the IGFBP-3 serum concentration (data not shown). In addition to its neuroendocrine role, LEP and LEPR regulate a wide range of biological processes, including mammary tumorigenesis ${ }^{5,7}$. LEP signaling promotes cell proliferation, differentiation, survival, migration, and invasion via the activation of downstream pathways, including the JAK2/STAT3, MAPK and PI3K/Akt pathways ${ }^{5,7}$. LEP crosstalk has also been reported with estrogen receptor alpha $(\mathrm{ER} \alpha)$ via ER $\alpha$ transactivation ${ }^{5}$ and the IGF-1 pathway through mTORC1 and STAT $5^{43}$. Based on these studies, our results suggest that the LEPR rs12059300 minor allele A could contribute to the reduction in BC risk. 
Notably, in addition to its modifying effect on the association between IGFBP-3 and BC risk, rs12059300 also showed an effect modification on the association between IGF-1 and the risk of BC $\left(P_{\text {interaction }}<0.05\right.$; adjusted $\left.P_{\text {interaction }}<0.20\right)$; however, this was not discussed in the IGF-1 section because associations inside strata were not statistically significant.

Finally, regarding the CARTPT SNP rs3846659, in women with the GG genotype, compared to the lowest IGFBP-3 serum concentration tertile, women in the highest tertile showed an increased BC risk, but this effect was lost in the presence of the minor allele C. In a previous work, our group found that rs3846659 was associated with the risk of premenopausal $\mathrm{BC}^{11}$. CARTPT encodes CART prepropeptide, which ends up generating multiple biologically active peptides that have a role in the regulation of appetite, energy balance, maintenance of body weight, reward and addiction, and the stress response ${ }^{44,45}$. CART is involved in the pituitary hormone secretion. Intracerebroventricular or peripheral administration of CART increases the GH concentration in rats $^{45}$, suggesting that CARTPT variants may modify the synthesis of IGF-1 and IGFBP-3. In our study, we did not find an association between the CARTPT rs3846659 SNP and the serum concentration of IGFBP-3 (data not shown). CART is expressed in primary and metastatic breast cancer; mediates ligand-independent activation of ERa through the MAPK pathway; and is an independent poor prognostic factor in ER-positive, lymph node-negative BC tumors ${ }^{4}$.

We were able to combine data from two population-based case-control studies conducted in the United States (4-Corner's Breast Cancer Study) and Mexico (Mexico Breast Cancer Study). Given that we used similar questionnaires for both studies, we were able to harmonize the lifestyle data. A tag SNP approach was used to characterize variation across candidate genes. This approach was implemented on a customized Illumina platform and included SNPs that were validated and considered to have a high probability of yielding results. We adjusted for multiple comparisons to identify SNPs with a relevant modifying effect, however spurious interactions cannot be excluded.

Regarding the limitations, only premenopausal women were included in this study. Previous large epidemiological studies have found statistically significant associations between IGF-1 and breast cancer only in premenopausal women; in postmenopausal women the associations were weaker and not statistically significant ${ }^{46-48}$. The percentage of indigenous ancestry was higher for the MBCS women than for those included in the 4-CBCS study. Therefore, we adjusted for the effect of both the study and indigenous ancestry in all multiple models. IGF- 1 and IGFBP-3 serum concentrations were measured in two different labs using two different assays. Some of the IGF-I and IGFBP-3 serum concentration discrepancies (less IGF-1 and more IGFBP-3 in the 4-CBCS than in the MBCS) between MBCS and 4-CBCS studies could be related to assay methodology or to differences in characteristics between women from both studies. More women in the 4-CBCS reported to consume alcohol than in the MBCS (controls $=41.8 \%$, cases $47.5 \%$; controls $=1.8 \%$, cases $=3.9 \%$, respectively). Alcohol consumption has shown to reduce IGF-I serum concentration and to increase IGFBP-3 serum concentration ${ }^{49}$. Although the IGF-1/IGBP-3 ratio may vary between studies $(0.03 \text { to } 0.10)^{50-52}$; the ratio remained constant in both cases and controls. In our study, the ratio in the 4-CBCS sample was 0.03 for both cases and controls, while in the MBCS sample it was 0.05 in both cases and controls. Therefore, we consider that it is unlikely that these discrepancies influenced our results. Although evidence suggest that carrying a BRCA mutation could be a potential confounder of the association between IGF-1 serum concentration and BC risk ${ }^{53,54}$, we did not exclude women with these mutations, because we have this information only for the MBCS women with breast cancer and not for controls. The prevalence of this mutation in the MBCS cases was $4.2 \%$, therefore adjusting for women with this mutation, could have not significantly altered our results. Finally, there is no information about the functional effect on protein expression caused by several of the SNPs analyzed in this study, thus limiting the biological comprehension of the observed interactions.

In summary, our data show for the first time that variants of energy homeostasis genes modify the effect of IGF-1 or IGFBP-3 serum concentration on BC risk in premenopausal women. We found that MBOAT4 and NPY SNPs modified the association between IGF-1 serum concentration and the risk of BC, and ADIPOQ, LEPR and CARTPT SNPs modified the association between IGFBP-3 and the risk of BC. Our data suggests that these genes could act as modifiers through their influence on known signaling pathways involved in BC development. Our results should be replicated in longitudinal studies and the molecular mechanisms responsible for the effect modifications that we found need to be further investigated. This study provides data that supports and highlights the importance of analyzing the interplay between multiple risk factors to contribute to a better understanding of breast cancer etiology and, in the future, improving risk prediction.

\section{Methods}

Study population. As part of the Breast Cancer Health Disparities study ${ }^{19}$, the study population included premenopausal women from the 4-Corner's Breast Cancer Study (4-CBCS) ${ }^{55}$ and the Mexico Breast Cancer Study (MBCS $)^{56}$. The study population for the present study was restricted to premenopausal women because in longitudinal studies, the association between the serum concentration of IGF-1 and the risk of BC has been observed in pre- but not postmenopausal women ${ }^{48,57}$. Briefly, the 4-CBCS included women living in Arizona, Colorado, New Mexico or Utah. Eligible cases were between 25 and 79 years old and were histologically confirmed between 1999 and $2004(n=2557)$, while controls $(n=2605)$ were selected from the target population and were frequency-matched to cases on the expected ethnicity and over a 5-year age range ${ }^{19}$. Interviews were performed by certified personnel ${ }^{58}$, blood was collected, and DNA was extracted for $79 \%$ of the participants; height, weight and waist and hip circumferences were obtained at interview ${ }^{19}$. For the present study, a subsample of 61 cases and 155 controls were included because they had fasting IGF-1 and IGFBP-3 measurements and were not receiving chemotherapy ${ }^{52}$. The MBCS included 1000 cases and 1074 controls between 35 and 69 years of age living in Monterrey, Veracruz and Mexico City for the past 5 years ${ }^{56}$. Eligible cases were women 
with BC histologically confirmed between January 2004 and December 2007. Controls were randomly selected from the catchment area of the 12 participating hospitals using a probabilistic multistage design. Controls were frequency-matched to cases, according to 5-year age groups, membership to a health care institution and place of residence. Interviews were performed by standardized personnel using a questionnaire based on the 4-CBCS. Blood was collected, and DNA was extracted from $91 \%$ of women. MBCS anthropometric measurements were obtained by standardized nurses at the hospitals. For this study, a random subsample of 204 cases and 282 controls who had serum IGF-1 and IGFBP-3 measurements was included.

Serum biomarkers. For both studies, serum levels of IGF-1 and IGFBP-3 were measured in fasting blood samples ${ }^{19,52}$. For the 4 -CBCS, IGF-1 and IGFBP-3 serum concentrations were measured at the Maine Center for Osteoporosis Research as described elsewhere ${ }^{59}$. Briefly, serum IGF-1 serum concentrations were determined using the IGF-1 (IGFBP-3 blocked) radioimmunoassay (American Laboratory Products Company (ALPCO) Windham, NH), while IGFBP-3 serum concentrations were ascertained using the "Active" IGFBP-3 IRMA kit (Diagnostic Systems Laboratories, Inc., Webster, Texas). All samples were tested in replicate, and the coefficient of variation ranged from 0.06 to $6.5 \%$ for IGF- 1 and 0.17 to $7.5 \%$ for IGFBP-3. For the MBCS, serum samples were frozen at temperatures between -20 and $-70{ }^{\circ} \mathrm{C}$ and processed no later than 4 weeks after the blood was collected. The IGF-1 and IGFBP-3 serum concentrations were determined using radioimmunoanalysis at Laboratorios Clínicos de Puebla, México (ISO 9001:2008).

Data harmonization. Data were harmonized as described in Slattery $(2012)^{19}$. Variables considered as potential confounders were age (years), genetic ancestry (\% Indigenous), body mass index (BMI, $\mathrm{kg} / \mathrm{m}^{2}$ ), energy intake (kcal/day), height $(\mathrm{cm})$, age at menarche (years), ever used oral contraceptives (no, yes), parity (nulliparous, 1 or 2, 3 or more), education (low, middle, high), study (4-CBCS, MBCS), first-degree family history of breast cancer (no, yes), ever consumed alcohol (no, yes), ever smoked cigarettes (no, yes) and IGFBP-3 serum concentration $(\mathrm{ng} / \mathrm{mL}$ ) when IGF-1 was the independent variable of interest, or IGF-1 serum concentration (ng/ $\mathrm{mL}$ ) when IGFBP-3 was the independent variable of interest. Weight used to calculate the BMI was measured by a nurse at the time of diagnosis for the MBCS and was either self-reported weight during the referent year or weight reported five years prior to diagnosis if the referent year weight was not available for the 4-CBCS. Height was measured at the time of interview.

Genetic data. DNA was derived from whole blood from both the 4-CBCS and the MBCS participants. Genotyping was conducted as part of the Breast Cancer Health Disparities Study ${ }^{19}$. European and Native American Ancestry were measured based on 104 ancestral informative markers. For this study, we evaluated 10 genes that regulate energy homeostasis: ADIPOQ (12 SNPs), CARTP (5 SNPs), CCK (4 SNPs), GHRL (8 SNPs), LEP (9 SNPs), LEPR (27 SNPs), MBOAT4 (1 SNP), MC4R (3 SNPs), NPY (4 SNPs), and POMC (5 SNPs). Supplementary Table $\mathrm{S} 1$ describes the 78 SNPs in detail.

Statistical analysis. A descriptive analysis of the study population was conducted. Means and standard deviations were used for continuous variables and percentages for categorical variables. Allele and genotype frequencies were obtained through direct counting. Hardy-Weinberg equilibrium (HWE) was tested by the exact test among control subjects. Comparison of genotype frequencies between cases and controls was adjusted for ancestry (\% Indigenous) by means of logistic regressions; $P$ values were obtained by using likelihood ratio tests. We used logistic regressions and likelihood ratio tests to compare ancestry adjusted genotype frequencies in control subjects from both populations. A $P$ value $<0.05$ was considered statistically significant. To evaluate the potential effect modification of the association between serum concentration of IGF-1 (tertiles) or IGFBP-3 (tertiles) and the risk of $\mathrm{BC}$ by energy homeostasis gene polymorphisms, multiple logistic regression models were performed. Associations with SNPs were assessed assuming a codominant model. Variables considered as potential confounders were described in the paragraph on data harmonization. Effect modification models are presented when $P$ for the interaction was lower than 0.10 . In addition, for multiple hypothesis testing correction Benjamini-Hochberg adjusted $P$ values were calculated for the number of SNPs tested per gene ${ }^{60}$; a threshold of 0.20 was considered significant ${ }^{61,62}$. The association between SNPs and IGF-1 and IGFBP-3 serum concentrations were analyzed. The Benjamini-Hochberg adjusted $P$ values were calculated for the number of SNPs tested per gene; a $P$ value $<0.05$ was considered statistically significant. Logistic regression analyses were performed using Stata12 (Stata Corporation, College Station, TX) and false discovery rate adjustments were performed using R version 3.6.2.

All participants signed informed written consent prior to participation; the study was approved by the Institutional Review Board for protection of human subjects at each institution.

Ethics approval. The study was approved by the Institutional Review Board for protection of human subjects at the National Institute of Public Health in Mexico and The University of Utah: "Comité de Ética en Investigación and Institutional Review Board, respectively" and was performed in accordance with the Declaration of Helsinki.

Consent to participate. Signed informed consent of all women participating in the study was obtained.

Consent for publication. All authors consent to the publication of this manuscript. 


\section{Data availability}

The datasets generated during and/or analyzed during the current study are not publicly available because it is not a requirement at our institution but are available from the corresponding author on reasonable request.

Received: 8 August 2021; Accepted: 17 December 2021

Published online: 03 February 2022

\section{References}

1. Khandekar, M. J., Cohen, P. \& Spiegelman, B. M. Molecular mechanisms of cancer development in obesity. Nat.. Rev. Cancer 11, 886-895. https://doi.org/10.1038/nrc3174 (2011).

2. Simpson, E. R. \& Brown, K. A. Minireview: Obesity and breast cancer: a tale of inflammation and dysregulated metabolism. Mol. Endocrinol. 27, 715-725. https://doi.org/10.1210/me.2013-1011 (2013).

3. Tilan, J. \& Kitlinska, J. Neuropeptide Y (NPY) in tumor growth and progression: Lessons learned from pediatric oncology. Neuropeptides 55, 55-66. https://doi.org/10.1016/j.npep.2015.10.005 (2016).

4. Brennan, D. J. et al. The cocaine- and amphetamine-regulated transcript mediates ligand-independent activation of ERalpha, and is an independent prognostic factor in node-negative breast cancer. Oncogene 31, 3483-3494. https://doi.org/10.1038/onc.2011. 519 (2012).

5. Ando, S. et al. Obesity, leptin and breast cancer: epidemiological evidence and proposed mechanisms. Cancers (Basel) 11, doi:https://doi.org/10.3390/cancers11010062 (2019).

6. Li, J. \& Han, X. Adipocytokines and breast cancer. Curr. Probl. Cancer 42, 208-214. https://doi.org/10.1016/j.currproblcancer. 2018.01.004 (2018).

7. Nalabolu, M. R., Palasamudram, K. \& Jamil, K. Adiponectin and leptin molecular actions and clinical significance in breast cancer. Int. J. Hematol. Oncol. Stem Cell Res. 8, 31-40 (2014).

8. Lin, T. C. \& Hsiao, M. Ghrelin and cancer progression. Biochim. Biophys. Acta 51-57, 2017. https://doi.org/10.1016/j.bbcan.2017. $02.002(1868)$.

9. Gahete, M. D. et al. Ghrelin gene products, receptors, and GOAT enzyme: biological and pathophysiological insight. J. Endocrinol. 220, R1-24. https://doi.org/10.1530/JOE-13-0391 (2014).

10. Thomas, R. P., Hellmich, M. R., Townsend, C. M. Jr. \& Evers, B. M. Role of gastrointestinal hormones in the proliferation of normal and neoplastic tissues. Endocr. Rev. 24, 571-599. https://doi.org/10.1210/er.2002-0028 (2003).

11. Slattery, M. L. et al. Energy homeostasis genes and breast cancer risk: The influence of ancestry, body size, and menopausal status, the breast cancer health disparities study. Cancer Epidemiol. 39, 1113-1122. https://doi.org/10.1016/j.canep.2015.08.012 (2015).

12. Allen, N. E. et al. A prospective study of serum insulin-like growth factor-I (IGF-I), IGF-II, IGF-binding protein-3 and breast cancer risk. Br. J. Cancer 92, 1283-1287. https://doi.org/10.1038/sj.bjc.6602471 (2005).

13. Martin, J. L. \& Baxter, R. C. Signalling pathways of insulin-like growth factors (IGFs) and IGF binding protein-3. Growth Factors 29, 235-244. https://doi.org/10.3109/08977194.2011.614237 (2011).

14. Weroha, S. J. \& Haluska, P. The insulin-like growth factor system in cancer. Endocrinol. Metab. Clin. North Am. 41, 335-350, vi, https://doi.org/10.1016/j.ecl.2012.04.014 (2012).

15. Bach, L. A. IGF-binding proteins. J. Mol. Endocrinol. 61, T11-T28. https://doi.org/10.1530/JME-17-0254 (2018)

16. Endogenous Hormones and Breast Cancer Collaborative Group, Key, T. J., Appleby, P. N., Reeves, G. K. \& Roddam, A. W. Insulinlike growth factor 1 (IGF1), IGF binding protein 3 (IGFBP3), and breast cancer risk: pooled individual data analysis of 17 prospective studies. Lancet Oncol. 11, 530-542, https://doi.org/10.1016/S1470-2045(10)70095-4 (2010).

17. Kaaks, R. et al. Insulin-like growth factor I and risk of breast cancer by age and hormone receptor status-A prospective study within the EPIC cohort. Int. J. Cancer 134, 2683-2690. https://doi.org/10.1002/ijc.28589 (2014).

18. Shi, R., Yu, H., McLarty, J. \& Glass, J. IGF-I and breast cancer: a meta-analysis. Int. J. Cancer 111, 418-423. https://doi.org/10.1002/ ijc.20233 (2004).

19. Slattery, M. L. et al. Genetic variation in genes involved in hormones, inflammation and energetic factors and breast cancer risk in an admixed population. Carcinogenesis 33, 1512-1521. https://doi.org/10.1093/carcin/bgs163 (2012).

20. Baglietto, L. et al. Circulating insulin-like growth factor-I and binding protein-3 and the risk of breast cancer. Cancer Epidemiol Biomarkers Prev 16, 763-768. https://doi.org/10.1158/1055-9965.EPI-06-0960 (2007).

21. Renehan, A. G. et al. Insulin-like growth factor (IGF)-I, IGF binding protein-3, and cancer risk: systematic review and metaregression analysis. Lancet 363, 1346-1353. https://doi.org/10.1016/S0140-6736(04)16044-3 (2004).

22. Al-Zahrani, A. et al. IGF1 and IGFBP3 tagging polymorphisms are associated with circulating levels of IGF1, IGFBP3 and risk of breast cancer. Hum. Mol. Genet. 15, 1-10. https://doi.org/10.1093/hmg/ddi398 (2006).

23. Ghoussaini, M. et al. Evidence that breast cancer risk at the $2 \mathrm{q} 35$ locus is mediated through IGFBP5 regulation. Nat. Commun. 4, 4999. https://doi.org/10.1038/ncomms5999 (2014).

24. Cevenini, A. et al. Molecular signatures of the insulin-like growth factor 1-mediated Epithelial-Mesenchymal transition in breast, lung and gastric cancers. Int. J. Mol. Sci. https://doi.org/10.3390/ijms19082411 (2018).

25. Shahjee, H. M. \& Bhattacharyya, N. Activation of various downstream signaling molecules by IGFBP-3. J. Cancer Ther. 5, 830-835. https://doi.org/10.4236/jct.2014.59091 (2014).

26. Pellatt, A. J. et al. Energy homeostasis genes and survival after breast cancer diagnosis: The Breast Cancer Health Disparities Study. Cancer Causes Control 27, 47-57. https://doi.org/10.1007/s10552-015-0681-6 (2016).

27. Wagner, K. et al. Polymorphisms in genes involved in GH1 release and their association with breast cancer risk. Carcinogenesis 27, 1867-1875. https://doi.org/10.1093/carcin/bgl036 (2006).

28. Dossus, L. et al. Polymorphisms of genes coding for ghrelin and its receptor in relation to anthropometry, circulating levels of IGF-I and IGFBP-3, and breast cancer risk: A case-control study nested within the European Prospective Investigation into Cancer and Nutrition (EPIC). Carcinogenesis 29, 1360-1366. https://doi.org/10.1093/carcin/bgn083 (2008).

29. da Cunha, P. A. et al. Interaction between obesity-related genes, FTO and MC4R, associated to an increase of breast cancer risk. Mol. Biol. Rep. 40, 6657-6664. https://doi.org/10.1007/s11033-013-2780-3 (2013).

30. Chopin, L. K., Seim, I., Walpole, C. M. \& Herington, A. C. The ghrelin axis-does it have an appetite for cancer progression?. Endocr. Rev. 33, 849-891. https://doi.org/10.1210/er.2011-1007 (2012).

31. Pabalan, N. A., Seim, I., Jarjanazi, H. \& Chopin, L. K. Associations between ghrelin and ghrelin receptor polymorphisms and cancer in Caucasian populations: a meta-analysis. BMC Genet. 15, 118. https://doi.org/10.1186/s12863-014-0118-3 (2014).

32. Gronberg, M. et al. Ghrelin is a prognostic marker and a potential therapeutic target in breast cancer. PLoS ONE 12, e0176059. https://doi.org/10.1371/journal.pone.0176059 (2017).

33. Peroski, M., Proudan, N., Grignol, G., Merchenthaler, I. \& Dudas, B. Corticotropin-releasing hormone (CRH)-immunoreactive (IR) axon varicosities target a subset of growth hormone-releasing hormone (GHRH)-IR neurons in the human hypothalamus. J. Chem. Neuroanat. 78, 119-124. https://doi.org/10.1016/j.jchemneu.2016.09.005 (2016).

34. Liu, L. et al. NPY1R is a novel peripheral blood marker predictive of metastasis and prognosis in breast cancer patients. Oncol. Lett. 9, 891-896. https://doi.org/10.3892/ol.2014.2721 (2015). 
35. Dhillon, P. K. et al. Common polymorphisms in the adiponectin and its receptor genes, adiponectin levels and the risk of prostate cancer. Cancer Epidemiol. Biomarkers Prev. 20, 2618-2627. https://doi.org/10.1158/1055-9965.EPI-11-0434 (2011).

36. Zhang, G. et al. ADIPOQ polymorphism rs182052 is associated with clear cell renal cell carcinoma. Cancer Sci. 106, 687-691. https://doi.org/10.1111/cas.12664 (2015).

37. Dobrzyn, K. et al. Adiponectin: A New Regulator of Female Reproductive System. Int. J. Endocrinol. 2018, 7965071. https://doi. org/10.1155/2018/7965071 (2018).

38. Rodriguez-Pacheco, F. et al. Regulation of pituitary cell function by adiponectin. Endocrinology 148, 401-410. https://doi.org/10. 1210/en.2006-1019 (2007).

39. Steyn, F. J. et al. Adiponectin regulate growth hormone secretion via adiponectin receptor mediated $\mathrm{Ca}(2+)$ signalling in rat somatotrophs in vitro. J. Neuroendocrinol. 21, 698-704. https://doi.org/10.1111/j.1365-2826.2009.01887.x (2009).

40. Psilopanagioti, A., Papadaki, H., Kranioti, E. F., Alexandrides, T. K. \& Varakis, J. N. Expression of adiponectin and adiponectin receptors in human pituitary gland and brain. Neuroendocrinology 89, 38-47. https://doi.org/10.1159/000151396 (2009).

41. Yousefi, M. et al. The methylation of the LEPR/LEPROT genotype at the promoter and body regions influence concentrations of leptin in girls and BMI at age 18 years if their mother smoked during pregnancy. Int. J. Mol. Epidemiol. Genet.. 4, 86-100 (2013).

42. Childs, G. V. et al. The somatotrope as a metabolic sensor: deletion of leptin receptors causes obesity. Endocrinology 152, 69-81. https://doi.org/10.1210/en.2010-0498 (2011).

43. Marwarha, G., Prasanthi, J. R., Schommer, J., Dasari, B. \& Ghribi, O. Molecular interplay between leptin, insulin-like growth factor-1, and beta-amyloid in organotypic slices from rabbit hippocampus. Mol. Neurodegener. 6, 41. https://doi.org/10.1186/ 1750-1326-6-41 (2011).

44. Rogge, G., Jones, D., Hubert, G. W., Lin, Y. \& Kuhar, M. J. CART peptides: regulators of body weight, reward and other functions. Nat. Rev. Neurosci. 9, 747-758. https://doi.org/10.1038/nrn2493 (2008).

45. Baranowska, B., Wolinska-Witort, E., Martynska, L., Chmielowska, M. \& Baranowska-Bik, A. Effects of cocaine-amphetamine regulated transcript (CART) on hormone release. Regul. Pept. 122, 55-59. https://doi.org/10.1016/j.regpep.2004.05.006 (2004).

46. findings from the UK Biobank.,. Tin Tin, S., Reeves, G. K. \& Key, T. J. Endogenous hormones and risk of invasive breast cancer in pre- and post-menopausal women. Br. J. Cancer 125, 126-134. https://doi.org/10.1038/s41416-021-01392-z (2021).

47. Murphy, N. et al. Insulin-like growth factor-1, insulin-like growth factor-binding protein-3, and breast cancer risk: observational and Mendelian randomization analyses with approximately 430000 women. Ann. Oncol. 31, 641-649. https://doi.org/10.1016/j. annonc.2020.01.066 (2020).

48. Hankinson, S. E. et al. Circulating concentrations of insulin-like growth factor-I and risk of breast cancer. Lancet 351, 1393-1396. https://doi.org/10.1016/S0140-6736(97)10384-1 (1998).

49. Lavigne, J. A. et al. Effects of alcohol on insulin-like growth factor I and insulin-like growth factor binding protein 3 in postmenopausal women. Am. J. Clin. Nutr. 81, 503-507. https://doi.org/10.1093/ajcn.81.2.503 (2005).

50. Rosendahl, A. H. et al. Pre- and postoperative circulating IGF-I, IGFBP-3, and IGFBP-7 levels in relation to endocrine treatment and breast cancer recurrence: A nested case-control study. Front. Oncol. 11, 626058. https://doi.org/10.3389/fonc.2021.626058 (2021).

51. Krajcik, R. A., Borofsky, N. D., Massardo, S. \& Orentreich, N. Insulin-like growth factor I (IGF-I), IGF-binding proteins, and breast cancer. Cancer Epidemiol. Biomarkers Prev. 11, 1566-1573 (2002).

52. Rollison, D. E. et al. Serum insulin-like growth factor (IGF)-1 and IGF binding protein-3 in relation to breast cancer among Hispanic and white, non-Hispanic women in the US Southwest. Breast Cancer Res. Treat 121, 661-669. https://doi.org/10.1007/ s10549-009-0609-5 (2010).

53. Pasanisi, P. et al. Serum levels of IGF-I and BRCA penetrance: a case control study in breast cancer families. Fam. Cancer 10, 521-528. https://doi.org/10.1007/s10689-011-9437-y (2011).

54. Kang, H. J. et al. BRCA1 negatively regulates IGF-1 expression through an estrogen-responsive element-like site. Cell Death Dis. 3, e336. https://doi.org/10.1038/cddis.2012.78 (2012).

55. Slattery, M. L. et al. Body size, weight change, fat distribution and breast cancer risk in Hispanic and non-Hispanic white women. Breast Cancer Res. Treat 102, 85-101. https://doi.org/10.1007/s10549-006-9292-y (2007).

56. Angeles-Llerenas, A. et al. Moderate physical activity and breast cancer risk: the effect of menopausal status. Cancer Causes Control 21, 577-586. https://doi.org/10.1007/s10552-009-9487-8 (2010).

57. Toniolo, P. et al. Serum insulin-like growth factor-I and breast cancer. Int. J. Cancer 88, 828-832 (2000).

58. Liu, K. et al. A study of the reliability and comparative validity of the cardia dietary history. Ethn. Dis. 4, 15-27 (1994).

59. Slattery, M. L. et al. Genetic, anthropometric, and lifestyle factors associated with IGF-1 and IGFBP-3 levels in Hispanic and nonHispanic white women. Cancer Causes Control 16, 1147-1157. https://doi.org/10.1007/s10552-005-0318-2 (2005).

60. Benjamini, Y. a. H., Y. Controlling the false discovery rate: A practical and powerful approach to multiple testing. J. R. Stat. Soc. Ser. B Methodol., 57, 289-300 (1995).

61. Hahn, M., Simons, C., Weijenberg, M. P. \& van den Brandt, P. A. Alcohol drinking, ADH1B and ADH1C genotypes and the risk of postmenopausal breast cancer by hormone receptor status: The Netherlands Cohort Study on diet and cancer. Carcinogenesis 39, 1342-1351. https://doi.org/10.1093/carcin/bgy101 (2018).

62. Geybels, M. S., van den Brandt, P. A., van Schooten, F. J. \& Verhage, B. A. Oxidative stress-related genetic variants, pro- and antioxidant intake and status, and advanced prostate cancer risk. Cancer Epidemiol. Biomarkers Prev 24, 178-186. https://doi.org/10. 1158/1055-9965.EPI-14-0968 (2015).

\section{Acknowledgements}

We would like to acknowledge María Felix Lazcano, Silvia Cardoso Muñoz and Libia Zulema Franco Velázquez for the Mexican study field work. The Mexico Breast Cancer Study was funded by Consejo Nacional de Ciencia y Tecnología (CONACyT) (SALUD-2002-C01-7462). The Breast Cancer Health Disparities Study was funded by Grant CA14002 from the National Cancer Institute.

\section{Author contributions}

G.T.-M., M.L.S. and A.A.L. conceived the study design. G.T.-M., M.L.S., E.Z., R.W., K.B.B. and L.H. conducted the data acquisition. G.T.-M., L.M.-M., L.G.-F.-R., R.R.-V., L.M.S.-Z., L.F.-L. and E.O.-P. prepared the data for analyses and carried out the analyses. G.T.-M. and R.R.-V. drafted the initial manuscript; M.L.S. and E.Z. undertook a thoroughly critical review of the manuscript. All authors revised and approved the final manuscript. None of the funding organizations had any role in the design and conduct of the study, or in the analysis of the data or in the writing of the manuscript. 


\section{Funding}

The Mexico Breast Cancer Study was funded by Consejo Nacional de Ciencia y Tecnología (CONACyT) (SALUD-2002-C01-7462). The Breast Cancer Health Disparities Study was funded by Grant CA14002 from the National Cancer Institute.

\section{Competing interests}

The authors declare no competing interests.

\section{Additional information}

Supplementary Information The online version contains supplementary material available at https://doi.org/ 10.1038/s41598-022-05496-1.

Correspondence and requests for materials should be addressed to G.T.-M.

Reprints and permissions information is available at www.nature.com/reprints.

Publisher's note Springer Nature remains neutral with regard to jurisdictional claims in published maps and institutional affiliations.

Open Access This article is licensed under a Creative Commons Attribution 4.0 International License, which permits use, sharing, adaptation, distribution and reproduction in any medium or
format, as long as you give appropriate credit to the original author(s) and the source, provide a link to the Creative Commons licence, and indicate if changes were made. The images or other third party material in this article are included in the article's Creative Commons licence, unless indicated otherwise in a credit line to the material. If material is not included in the article's Creative Commons licence and your intended use is not permitted by statutory regulation or exceeds the permitted use, you will need to obtain permission directly from the copyright holder. To view a copy of this licence, visit http://creativecommons.org/licenses/by/4.0/.

(C) The Author(s) 2022 